\title{
Changes in the Activity of Osteoblast Like Cells with Sol-Gel Derived Hydroxyapatite and Zirconia Nanocoatings
}

\author{
K.Lewis $^{1}$ a, S. M. Valenzuela ${ }^{2}$, B. Ben-Nissan ${ }^{1}$ \\ ${ }^{1}$ Department of Chemistry Materials and Forensic Science, ${ }^{2}$ Department of Medical and Molecular \\ Biosciences, University of Technology Sydney, PO Box 123 Broadway NSW 2007 Australia \\ aKanthi.Lewis@uts.edu.au
}

Keywords: bone, sol-gel, hyroxyapatite, zirconia, nanocoatings, osteoblast

\begin{abstract}
When producing implant materials, achievement of optimal bioactivity and biocompatibility are essential. Nanocoatings can provide an efficient cost effective way to alter the interactions of the implant material with its destined "host" environment. Nanocoatings of sol-gel derived carbonated hydroxyapatite (HAp) and zirconia were produced in this study. The surfaces were characterised by Fourier transform infrared spectroscopy (FTIR) and light microscopy. Cell adhesion, proliferation and viability, as well as expression of alkaline phosphatase (ALP is an indicator of bone formation) were assessed as indicators of biocompatibility. Our results have shown that sol-gel derived nano crystalline HAp acts as an ideal surface for implant coatings.
\end{abstract}

\section{Introduction}

Implant materials are being increasingly designed to provide optimal pore structure for osteointegration, and a subsequent increase in the bone to implant bond [1]. Increases in the bioactivity have been achieved by the creation of micro and nano-textured surfaces. While these provide a surface conducive to bone ingrowth, the material used needs more study with regard to the effect on cell activity and the changes which occur to intracellular processes in the presence of these implant materials. This work reports a series of characterisation studies to observe the changes occurring to 2 osteoblast cell lines (Mg63 and Saos-2) when cultured on sol-gel derived nanocoated surfaces, which can be used as implant coatings.

Sol-gel coating is described as any chemical procedure capable of producing ceramic oxides, nonoxides and mixed oxides from solutions. The advantages of the sol-gel technique are numerous. It results in a stoichiometric, homogeneous and pure coating due to mixing on the molecular scale; reduced firing temperatures due to small particle sizes with high surface areas; it has the ability to produce uniform fine-grained structures; the use of different chemical routes (alkoxide or aqueousbased); and their ease of application to complex shapes with a range of coating techniques: dip, spin, and spray coating.

\section{Materials and Methods}

Preparation and Characterisation of Coated Surfaces. Glass cover slips are spin with an alkoxide-derived sol-gel carbonate hydroxyapatite (CHAp), and sol gel-zirconia oxide solutions followed by firing to $550^{\circ} \mathrm{C}$ [2]. Characterisation of the surfaces is conducted using light microscopy as well as Fourier transform infrared spectroscopy (FTIR) All FTIR data has been obtained using a Nicolet magna IRE 760, measurements were conducted using diffuse powder reflectance, with a $\mathrm{KBr}$ reference. Thickness of the coatings is dependent on the number of layers and is varied between $70-300 \mathrm{~nm}$.

Osteoblast Cell Culturing and Bio-Assays. Human osteosarcoma cell lines Mg63 and Saos-2 (ATCC, USA) were plated onto coated glass coverslips as well as uncoated glass coverslip as a control. The control coverslips are also fired to remove variations between samples due to possible bioactivity changes of the glass with firing. After plating onto the surfaces, Mg63 and Saos-2 cells were grown at intervals of 1, 3, 5, and 7 days, in Eagles Minimum Essential Media (Sigma) with 
10\% fetal bovine serum (FBS)(Sigma) and McCoys modified media SA (Sigma) with 15\% FBS respectively, as per the supplied protocol. The cell samples were then assessed for cell proliferation and viability using propidium iodide exclusion staining.

A three hour adhesion assay was also conducted, by plating the cells on each surface and removing the supernatant after 3 hours. Propidium iodide (PI) was added to each of the cell surfaces, and fluorescence readings were obtained after freeze thawing [3]. Cell numbers were determined via a standard curve. Cell morphology was examined by light microscopy. The production of serum Alkaline Phosphatase (ALP) was measured via absorbance assays using p-nitrophenyl phosphate (Sigma, Australia), based on the following equation;

$$
\begin{aligned}
& \text { ALP } \\
& \text { p-Nitrophenyl phosphate }+\mathrm{H} 20 \quad-\text { ? } \quad \text { p-Nitrophenol (pNP) }+\mathrm{H}_{3} \mathrm{PO}_{4}
\end{aligned}
$$

As the reaction procedes the solution changes from colourless to yellow, and the appearance of $p$ nitrophenol is measured spectrophotometrically at $405 \mathrm{~nm}$. Samples are incubated for thirty minutes and one unit of phosphatase activity is defined as the amount of enzyme that hydrolyzes 1 micromole of pNPP per litre.

Each experiment is conducted with a minimum of 4 repeats, and each reading is repeated 3 times for instrument precision, average results are used for analysis, and any outliers are excluded using Q-test at a 95\% confidence interval.

\section{Results and Discussion}

FTIR studies showed that HAp coatings were pure carbonated hydroxyapatite with a nanocrystalline structure.

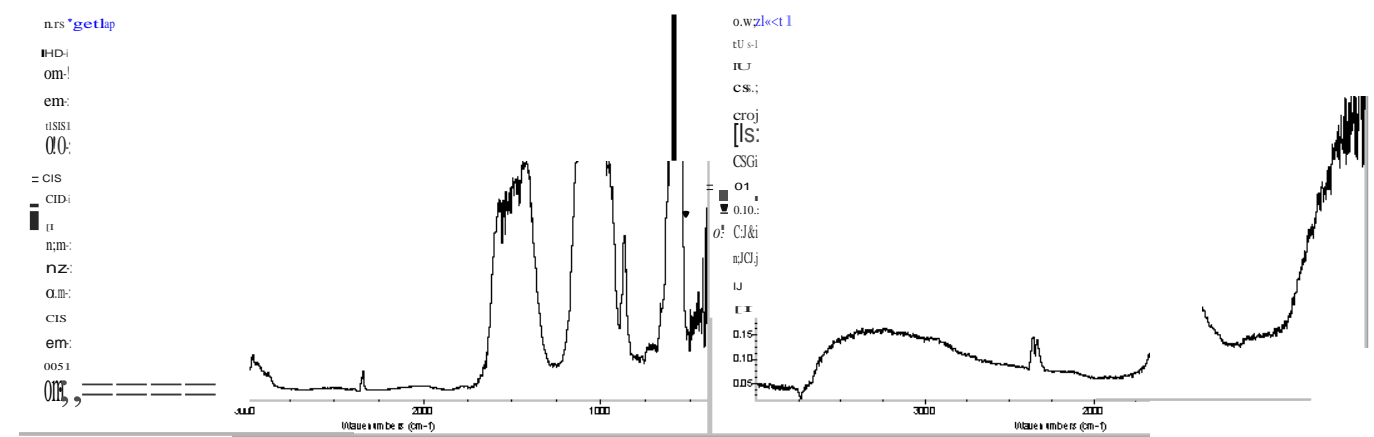

Figure 1 FTIR data a) sol-gel HAp and b) sol-gel $\mathrm{ZrO}_{2}$

The FTIR data in Figure 1 confirms that organic material from the solution has been removed during heating to $550^{\circ} \mathrm{C}$. This can be seen as only peaks which can be assigned to the hydroxyapatite are present in the spectrum. The small band at $3000 \mathrm{~cm}^{\cdot}$ can be assigned to the $0-\mathrm{H}$ stretch in the crystal lattice. The small crystallite size characteristic of nanosized crystals is a contributing factor to the resolution of the phosphate peaks. The splitting of the bands between 1100-1000 $\mathrm{cm} \cdot{ }^{1}$ and 600-550 cm. ${ }^{1}$ will differ due to crystallite size [4].

The carbonate bands are observed at 873, 1417 and $1475 \mathrm{~cm}^{\cdot}{ }^{4}$ [4]. Characteristic of a carbonate apatite. The incorporation of carbonate has been shown to increase the bioactivity of Hydroxyapatite [5].

FTIR absorption spectra is typical of an alkali treated zirconia. The absorption peaks at 418 and 745 em 1 in both the samples are of typical monoclinic zirconia, in addition two peaks appear at 1116 and $1156 \mathrm{~cm} \_1$ which have been reported in nanocrystaline $\mathrm{Zr02}$ powders produced [5]. There is also a broad hydroxyl band above 3000 em 1 clearly showing some water remaining in the product. 
As water absorbs very strongly, this band although broad, has a low intensity, indicating the water content is minimal.
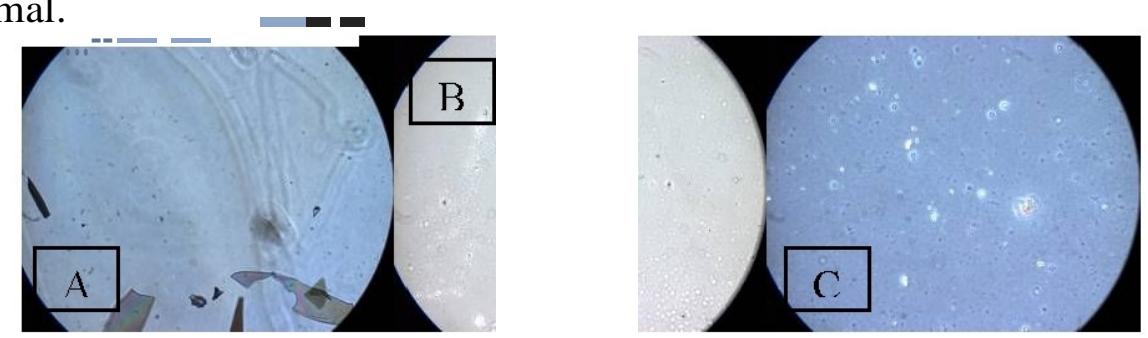

Figure 2. Light microscope images of the coatings produced A) zirconia, B) and C) Hydroxyapatite coatings

In Figure 2, the images a) and b) show the zirconia coatings, the coating of the surface is fairly uniform, and although some cracking is observed around the edges of the coverslip the remainder was free from cracks. The hydroxyapatite coatings did not show any of the cracks observed in the zirconia coatings.

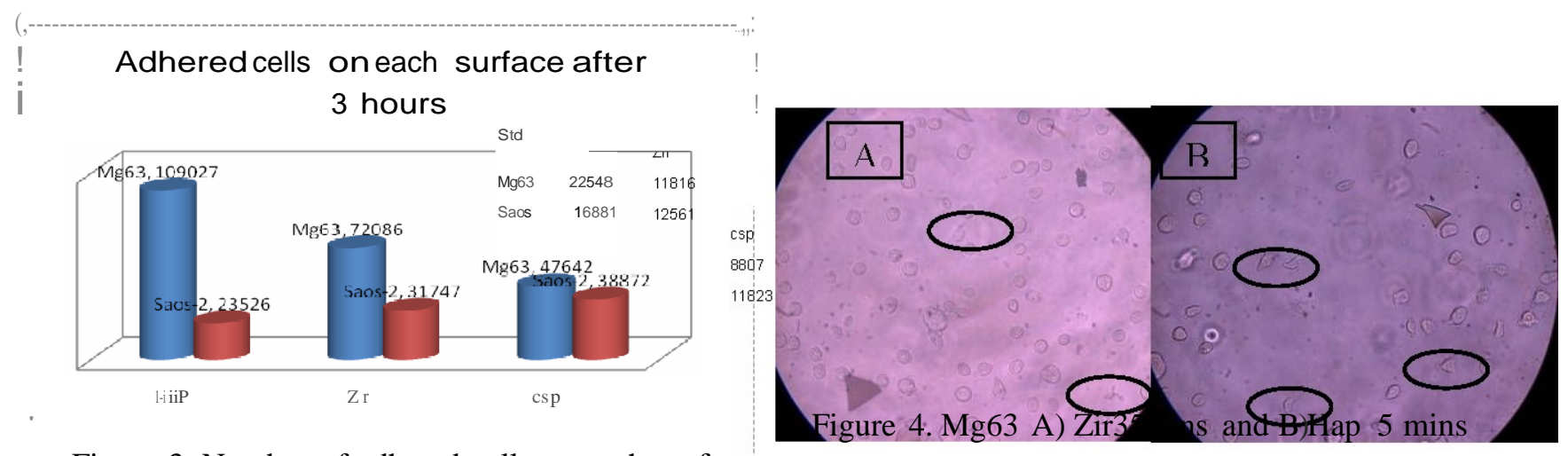

Figure 3. Number of adhered cells on each surface

In Figures 3 and 4 Mg63 cell line demonstrated a dramatic increase in adherence on hydroxyapatite. Figure 4 (b) shows the number of cells adhered and already elongating on the HAp surface (indicated by black outline) at 5 minutes. However at 35 minutes on the zirconia coating, this level of adhesion is not observed. (The cells starting to adhere are circled in black). This is also seen from the adhesion assay, which shows an increase in adhered cell number for both the HAp and Zirconia coatings. The largest increase was on the hydroxyapatite coating. The Saos-2 cells, did not prefer the coated substrates for adhesion, however the differences between attached cell number on each coating are much smaller.

In order to confirm that cytotoxicity was not the reason for the reduced number of cells observed on the coatings, a test of the total number of dead cells in each well was determined. The control coverslips also showed a number of dead cells over time, however to demonstrate the coating effect on cell death, the cell numbers have been compared to that ofthe control (Fig. 5).

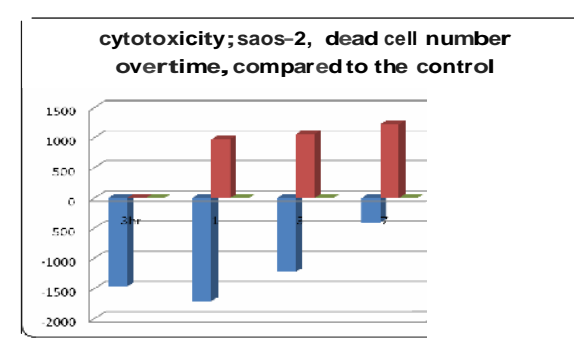

Figure 5. Dead cell number compared to the control at time intervals 3 hours, 1, 3, 5, and 7 days 
The cells were plated at $1.5 \times 10^{5}$ cells per ml, with the number of dead cells measured before freeze thawing carried out at each time interval using PI. It can be seen that far less dead cells were present when the cells were grown on Hydroxyapatite compared to the control, indicating that the although these cells are not adhering as quickly, they are infact not dieing. As the time interval increases the differenece between the hydroxyapatite and the control decreases. The zirconia coating shows an increase in the cell death, given the growth over time, this is however a differenece of much less than $1 \%$ of the total cell number. Given that there is also a decrease in the proliferation of Saos-2 on this surface (Fig. 6), further work needs to be conducted to determine effects on cell cytotoxicity.

The proliferation assay also demonstrated that the Mg63 cell line shows a slight preference for growth on the hydroxyapatite surface, however this change is minimal. Although the Mg63 cell line showed an increased adhesion on zirconia compared to the control, this did not however lead to an increase in overall cell proliferation.

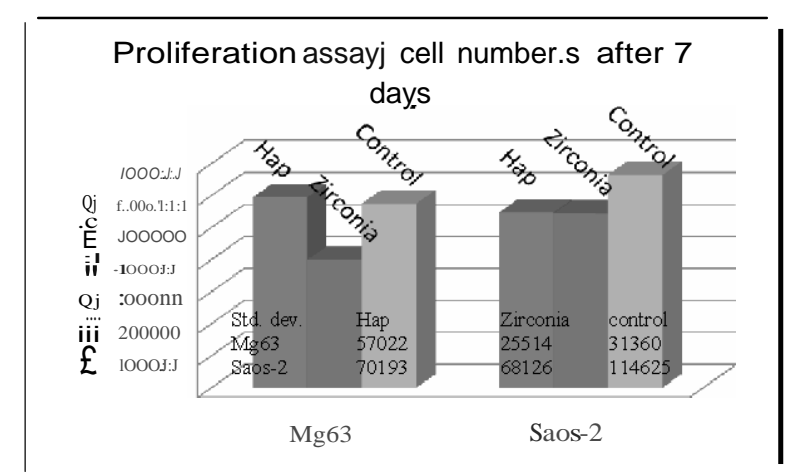

Figure 6. cell number after 7 days, determined by PI assay

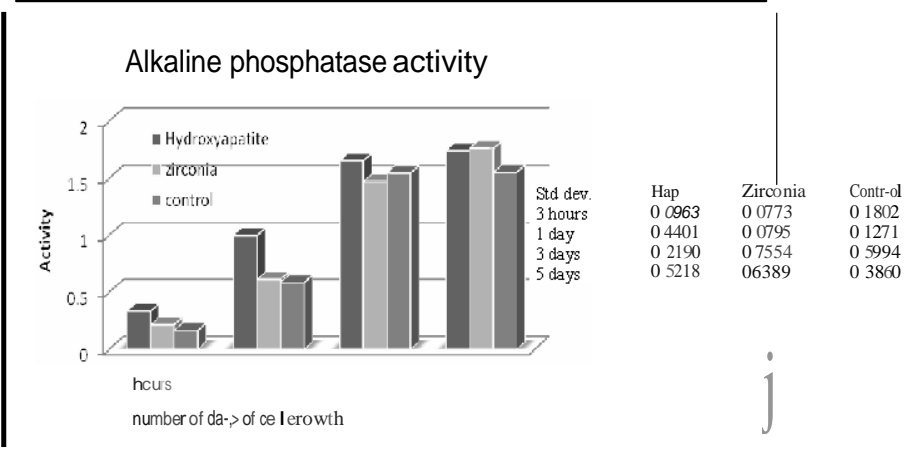

Figure 7. Alkaline phosphatase activity ofMg63 Cell line ()lg/min/L)

Investigation of the alkaline phosphatase activity also indicated increased levels for the cells grown on hydroxyapatite compared to the control and the zirconia surface. This is possibly due to the increased cell number, and the decrease in adhesion time for the cells. The cells plated on zirconia showed a lower ALP activity for all timepoints, except the final day. Given the higher number of dead cells this may be due to the breakdown of cells therefore releasing intracellular ALP into the supernatant.

\section{Conclusion}

Hydroxyapatite was the preferred surface for the growth of the 2 cell lines examined. It was found to have a significant effect on the Mg63 cell line, which demonstrated increased adhesion, proliferation and ALP activity. Altering the surface coating was found to have little to no effect on the Saos-2 cell line, with only a slight increase in the cell viability.

\section{References}

[1] Zreiqat, H., et al. Key Engineering Materials, 2005. 284-246: p. 541-554.

[2] Chai CS, et. al, B., J. Mat. Sci. Mat. Med (1999) 10, 465 -649

[3] Dengler, et.al. Anticancer drugs 19956 pp 552-532

[4] Lewis, K., et. al. (2005), Journal of the Australasian Ceramic Society, 41, 2, p52-55.

[5] R. Z. LeGeros, Progress in Crystal Growth and Characterization of Materials, 4, p1-45. (1981)

[6] Southon, P. PhD thesis. University of Technology Sydney (2000). 